\title{
Redes empresariales locales y su incidencia en la innovación de la empresa
}

\author{
Becerra Rodríguez, Fredy* \\ Serna Gómez, Héctor Mauricio**
}

\section{Resumen}

La importancia de las actividades de investigación y desarrollo -I\&D- y de la innovación es reconocida en la literatura, dado que éstas son las que permiten a la empresa generar aspectos diferenciadores. Este trabajo tiene como objetivo identificar la incidencia de los vínculos entre empresas y otras instituciones a nivel local para el desarrollo de innovación y actividades de I\&D en la empresa en la ciudad de Manizales, Colombia, específicamente se analizan los vínculos entre empresas para actividades de I\&D y la transferencia de información técnica y tecnológica. Para el cumplimiento de los objetivos se recopiló información de 246 empresas, las cuales son el total de la población del clúster textil de la misma ciudad, para la contrastación empírica se utilizaron modelos logit. Como resultados se evidencia un bajo nivel de actividades de I\&D e innovación en la empresa y se establece que la existencia de vínculos para actividades de I\&D entre empresas incrementan la probabilidad en un $49 \%$ de generar innovación en la empresa y en un $80 \%$ las actividades de I\&D en la empresa. Se concluye que los vínculos entre empresas fortalecen las actividades de I \& D en mayor proporción que las que no están vinculadas, asimismo esta vinculación repercute directamente en la competitividad del sector estudiado.

Palabras clave: Redes empresariales, Investigación y desarrollo (I\&D), Transferencia tecnológica, sector textil.

\section{Recibido: 30-05-11. Aceptado: 09-11-11}

* Doctor en administración, Universidad San Pablo CEU (Madrid - España). Ingeniero Industrial, Universidad Nacional de Colombia (Manizales - Colombia). Profesor Asociado Universidad Nacional de Colombia (Manizales - Colombia). Profesor Asociado Universidad de Manizales (Manizales - Colombia). e-mail: fbecerraro@unal.edu.co.

** Administrador de empresas, Universidad Nacional de Colombia (Manizales - Colombia). Investigador programa Virginia Gutiérrez de Pineda - Colciencias. e-mail: hmsernag@ bt. unal.edu.co. 


\title{
Local Entrepreneurial Networks and their Effect on Innovation in Companies
}

\begin{abstract}
The importance of research and development activities - R \& D- and innovation is recognized in the literature, given that they are what allow a company to generate differentiating aspects. The objective of this study is to identify the effect that connections between companies and other institutions on the local level have on the development of innovation and R\&D activities in companies in the city of Manizales, Colombia. Specifically, connections between companies for R\&D activities and the transfer of technical and technological information are analyzed. To fulfill the objectives, information was collected from 246 companies, the total population for the textile cluster in the same city. For empirical contrasting, logit models were used. Results showed a low level of R\&D activities and innovation in the companies, and it was established that the existence of connections for R\&D activity among companies increase the probability by $49 \%$ of generating innovation in the company and in $80 \%$ of the company's R\&D activities. Conclusions are that companies with connections among themselves strengthen R\&D activities to a greater proportion than those that have no interconnections; likewise, the connection has direct repercussions on competitiveness in the sector under study.
\end{abstract}

Keywords: Entrepreneurial networks, research and development (R\&D), technological transfer, textile sector.

\section{Introducción}

La importancia que tienen las actividades de I\&D y la innovación en el desempeño de la empresa es ampliamente reconocida en la literatura (Damanpour, 1991; Damanpour \& Gopalakrishnan, 1998; Wolfe, 1994; OCDE, 1999 a y b; Damanpour \& Gopalakrishnan, 2001; Jamrog et al., 2006; López, 2006; Muscio, 2006), dado que éstas le permiten a la empresa incrementar sus capacidades técnicas, tecnológicas, organizativas y de gestión (Taylor et al., 2008). Aunque los estudios sobre investigación, desarrollo e innovación -I\&D\&I- han estado centrados a nivel de la empresa, se reconoce como determinante de ésta la importancia que el entorno tiene para su desarrollo dentro de la empresa (Seufert et al., 1999; Camisón et al., 2003).

Las relaciones entre empresas y otros agentes son un factor determinante en la construcción y transferencia de conocimientos, y refuerzan los mecanismos de aprendizaje de las empresas y su capacidad innovadora (Capello, 2002; Belusi \& Pilotti, 2002; Capello \& Faggian, 2005; Caniëls \& Romijn, 2003; Arvanitis et al., 2005, 2007). No obstante, y pese a reconocer las relaciones como determinante de la innovación en la empresa (Seufert et al., 1999; Camisón et al., 2003), la literatura es escasa en estudios que aborden el fenómeno relacional en los sistemas productivos locales (Capello, 2002), probablemente por la intangibilidad (Capello y Faggian, 2005) del fenómeno, muy notorio en economías emergentes o en vías de desarrollo, como la Colombiana.

En general, se puede establecer que las empresas crean y desarrollan capacidades para la innovación en la medida en que aprovechan sus propios recursos y 
los de otros agentes que las rodean (competidores, proveedores, clientes, instituciones, entre otros), en particular en contextos donde el acceso a recursos es muy limitado, como es el caso de los países en desarrollo y de economías emergentes como la colombiana. Los vínculos entre empresas y con otras instituciones, dentro de sistemas productivos localizados particularmente las redes empresariales (Becerra y Álvarez 2011), en función de mejorar el desempeño de las empresas, implican crear ventajas competitivas a partir de los recursos endógenos propios de la empresa y de la localidad donde éstas se albergan a partir de la innovación entendida como resultado (OCDE/EUROSTAT, 2005), como proceso (Slappendel, 1996; Wolfe, 1994) y como aquellas innovaciones que trascienden la empresa para situarse en lo socio económico (Becerra y Naranjo, 2008).

En la perspectiva anotada, en Colombia se presenta ausencia de estudios sobre la relación entre empresas, (Cardona et. al. 2006), sólo en el año 2008 el Consejo Privado de Competitividad CPC- vislumbra de manera preliminar el desarrollo de las relaciones empresariales a partir de la conformación de clusters en las regiones (CPC, 2008), esto lleva a que algunos académicos realicen descripciones que permitan identificar encadenamientos empresariales en Colombia. Algunos trabajos han analizado la existencia de vínculos entre empresas de un sector en una región específica (Pietrobelli \& Olarte, 2002), otros han analizado la relación capital producto por empresa perteneciente a un sector en una región específica y su relación con el costo laboral unitario, esto con el fin de identifi- car la existencia de clusters (Cardona \& Cano, 2004), y otros estudios han desarrollado taxonomías para analizar la capacidad innovadora de la empresa dentro de una red empresarial (Becerra \& Naranjo, 2008). De acuerdo a lo anterior, el trabajo reviste de importancia ya que analiza aspectos que han sido poco estudiados en el contexto colombiano como es el caso de las redes empresariales, y además aborda el fenómeno relacional entre empresas aspecto que es poco tratado en la literatura (Capello, 2002).

El trabajo se basa en los resultados obtenidos por el estudio "Dinámica interna de microclusters en el Departamento de Caldas (confecciones, salud, turismo, herramientas agrícolas, electrodomésticos, muebles de madera)" ejecutado entre los años 2004 y 2005, datos que todavía son relevantes, ya que la estructura empresarial no ha tenido modificaciones sustanciales y los estudios sobre dichos sectores no abordan las perspectiva que tiene el presente trabajo que aún sigue siendo referente para la comprensión de las relaciones entre empresas y otras instituciones en función de la innovación y la I\&D en la empresa tanto para el país como para la región donde se ha realizado la investigación.

En este estudio se utilizó un cuestionario estructurado, previa una prueba piloto con la que se eliminaron ambigüedades y se generó validez del mismo (Mahemba \& De Bruijn, 2003). El cuestionario fue aplicado mediante entrevistas personales a los gerentes o dueños. El estudio indagó por cinco factores en las empresas que componen el cluster: el primero son aspectos comerciales y de mercado; segundo aspectos de capital huma- 
no y trabajo; tercero aspectos de ciencia, tecnología e innovación; cuarto aspectos económicos y financieros de la empresa; y el quinto aspecto son relaciones informales. El último factor es el que analiza las relaciones entre empresas e instituciones en función de la innovación y las actividades de I\&D, y el cual es objeto de análisis del presente trabajo.

La importancia de este trabajo en el cluster textil- confección del departamento de caldas se justifica en varias circunstancias:

- Desde el punto de vista nacional, El Ministerio de Comercio, Industria y Turismo - MCIT colombiano ha considerado al sector como fundamental en la generación de empleo y como uno de los sectores de clase mundial, dado que participa con el $22 \%$ del empleo manufacturero del país y con el $5 \%$ del producto interno bruto - PIB (MCIT, 2009) y según PROEXPORT (2009), tanto el crecimiento del sector como su participación en las exportaciones que es del orden del $5 \%$, implica mejoras en productividad, tecnología e innovación dentro de las empresas de confecciones.

- Desde el punto de vista regional el cluster está constituido en su mayoría por micro y pequeñas empresas con poca capacidad para desarrollar innovaciones, y con dificultades para aprovechar oportunidades del medio, en particular las relaciones con agentes de investigación y universidades en función de la innovación y la tecnología, no obstante es un sector que en el período 2002 - 2005 presentó una balanza comercial positiva (Departamento Nacional de Planeación - DNP. 2007) y dentro de la economía de Caldas, el sector textil-confección es uno de los seis sectores de la industria manufacturera que concentran el $13 \%$ del PIB departamental y el $62 \%$ del empleo industrial (DNP, 2010). Recientemente el sector ha sido considerado una de las apuestas productivas por parte de agentes gubernamentales y no gubernamentales de los departamentos que conforman la región del Eje Cafetero ${ }^{1}$ dado que "concentra unas $1.400 \mathrm{em}$ presas de la confección, lo cual representa aproximadamente el $20 \%$ de empresas y empleos del sector en el país. De acuerdo a una encuesta reciente, el 95\% de las empresas confeccionistas de la zona son micro, pequeñas y medianas empresas (MIPYMES), así el $59 \%$ son microempresas, el $19 \%$ pequeñas empresas y el $17 \%$ medianas empresas, y el 5\% restante grandes empresas" (BID, 2002, 12).

Los aspectos mencionados permiten plantear que los resultados del presente trabajo aportan información sustancial para el logro del objetivo y ofrecen elementos para una comprensión sobre la estructura de la red y su dinámica para el desarrollo de capacidades de innovación en las empresas que la componen, así como dar un marco de referencia para 
la política pública en el departamento de Caldas.

El cluster textil de la ciudad de Manizales, departamento de Caldas, Colombia lo componen 246 empresas distribuidas en los eslabones de la cadena $-13 \%$ proveedores, $39 \%$ fabricantes, y $48 \%$ distribuidores-, para el estudio se recopiló información del total de los establecimientos del cluster, utilizando para ello las bases de datos de Cámara de Comercio de Manizales, Asociación Nacional de Industriales (ANDI) y Asociación Colombiana de pequeños industriales (ACOPI), y triangulando la información para consolidar la población estudiada.

\section{El concepto de innovación y tipos de innovación}

El concepto de innovación tiene sus orígenes en los trabajos de Schumpeter (1997) quien plantea que la innovación se da cuando se introduce un nuevo bien, cuando se realiza modificaciones en la calidad de un producto, cuando se desarrolla un nuevo método de producción, cuando se abre un nuevo mercado o cuando se conquista una nueva fuente de aprovisionamiento (Schumpeter, 1997).

A partir de esta definición algunos autores han planteado taxonomías realizando distinciones entre los tipos de innovación, Damanpour (1987) distingue entre innovaciones tecnológicas y administrativas y Gopalakrishnan \& Damanpour (1998) de acuerdo con el grado de innovación las clasifican en radicales e incrementales. Pero es con el manual de Oslo que se trasciende la innovación asociada básicamente a productos y procesos hacia áreas blandas como la comercializa- ción y la organización, sin ser descuidados los planteamientos de los autores anteriores, en este escrito se define la innovación como "la introducción de un nuevo, o significativamente mejorado, producto (bien o servicio), de un proceso, de un nuevo método de comercialización o de un nuevo método organizacional, en las prácticas internas de la empresa, la organización del lugar de trabajo o las relaciones exteriores" (OCDE/EUROSTAT, 2005, p. 56).

Esta definición además de integrar las distintas nociones presentes en la literatura sobre innovación, incluye y destaca las relaciones exteriores de la empresa, que implícitamente aluden al capital relacional que se configura mediante relaciones con otras empresas e instituciones (Capello, 2002; Capello y Faggian, 2005), centro de atención en este trabajo.

Además de la importancia que ha tenido, en la literatura, la innovación como resultado (Damanpour, 1987; Damanpour et al., 1989; West \& Farr, 1990; Hage, 1999; Damapour \& Gopalakrishnan, 2001; Jaskyte, 2005; OCDE/ EUROSTAT, 2005), se encuentra el interés que suscita el proceso mediante el cual las nuevas ideas, los objetos y las prácticas son creadas y desarrolladas o reinventadas (Slappendel, 1996), o en términos de Wolfe (1994) como las innovaciones surgen, se desarrollan y terminan, y actividad que denomina proceso de innovación. Al respecto, algunos autores hacen referencia a las fases que suceden desde que una invención es realizada hasta que alcanza su comercialización, siendo éstas tareas de investigación (básica y aplicada), desarrollo de prototipos, modelos, adquisición de tec- 
nología, y algunas etapas de la ingeniería del proyecto antes de ser implementado (OCDE/FECYT, 2002, OCDE/EUROSTAT, 2005; Rammer \& Schmiele, 2009).

Es de resaltar que las fases que suceden en un proceso de innovación se dan generalmente en un proceso interactivo (Edwards, 2003), y en el cual intervienen diversos actores, pudiendo ser actores de la organización o actores externos a ésta, a este tipo de intervenciones Edwards (2003) las denomina como actividades intra-empresariales e inter-empresariales, la primera se refiere al proceso de innovación generado por actores de la misma empresa, y la segunda se refiere al proceso de innovación generado por actores de diferentes empresas (Edwards, 2003).

\section{Redes empresariales: ambientes para la i\&d\&i}

La innovación y las actividades de innovación ocurren dentro de la empresa, pero más allá, se presentan en los ambientes donde se encuentran localizadas, aspecto que ha sido abordado en la literatura en diferentes nociones: cluster, distritos industriales, redes horizontales y verticales. Estos elementos se pueden generalizar bajo el concepto de red empresarial localizada (Becerra, 2008), dado que permiten agrupar tres perspectivas: localización y la concentración de las empresas -aglomeración empresarial-; y aspectos transaccionales.

Desde la primera perspectiva algunos autores como Baptista \& Swann (1998), Feldman \& Audretsch (1999) y Alecke et al. (2006) dan evidencia de que las empresas aglomeradas en una locali- dad son más proclives a la innovación que las que no lo están (Beaudry \& Breschi, 2003). Esto se da porque en los clusters y distritos industriales existe un mercado laboral de calidad, con experiencia, cualificación, con alta movilidad laboral (Hu et al., 2005) y con canales de comunicación a través de las redes interpersonales (Power \& Lundmark, 2004), lo cual, conlleva a que entre empresas se favorezcan de la transferencia de conocimientos tecnológicos tácitos y no codificados (Evangelista et al., 1997).

Desde la segunda perspectiva algunos autores analizan como las transacciones permiten generar innovación desde la red localizada (Hauknes, 1999), especificamente esta perspectiva analiza la existencia de transferencia de tecnología (Lagendijk \& Charles, 1999; Nadvi, 1999; Black et al.,2004, Arvanitis et al., 2007) y la transferencia de conocimiento y de información entre empresas (Callejón, 1998; Black et al., 2004; Caniëls \& Romijn, 2005; Alecke et al., 2006); la primera se da generalmente desde un aspecto comercial (Hauknes, 1999), mientras que la segunda se da a partir de la existencia de capital intelectual (Pöyhönen \& Smedlund, 2004; Hervás \& Dalmau, 2006) o de capital relacional (Capello, 2002; Capello \& Faggian, 2005) en la red localizada.

En los trabajos empíricos hay evidencian, desde las dos perspectivas, del papel que juegan agentes gubernamentales y no gubernamentales (Lagendijk \& Charles, 1999, Becheikh et al., 2006), la incidencia de la cercanía geográfica (Feldman \& Audretsch, 1999) y la incidencia de la existencia de una relación comercial sobre la innovación (Hauknes, 1999), pero es importante establecer que 
los estudios que analizan la transferencia de información y de conocimientos vista desde el capital relacional y el capital intelectual demuestran aspectos de intangibilidad para abordar el fenómeno y establecen que desde esta perspectiva se puede generar mayor comprensión del fenómeno de las redes empresariales locales y la innovación en la empresa (Capello, 2002; Capello \& Faggian, 2005).

Como se desprende de la literatura referenciada, las redes empresariales constituyen un ambiente favorable para la innovación empresarial y la transferencia de información y conocimiento tecnológico entre las empresas, en especial para las pequeñas y medianas empresas -PYMES- que acceden con mayor fluidez al desarrollo del conocimiento, amplían sus capacidades empresariales para generar procesos de innovación, y mejoran los resultados en las innovaciones de procesos y productos así como las administrativas (Jensen, 1996; Feldman \& Audretsch, 1999; Feser, \& Bergman, 2000; Nassimbeni, 2003; Alonso-Villar et al., 2004; Mella et al., 2005; Eraydin \& Amartli-Köroglu, 2005; Novick \& Carrillo, 2007).

Con el objeto de contribuir a la discusión sobre las redes empresariales localizadas, el presente trabajo analiza específicamente las relaciones ente empresas dentro de las redes localizadas desde dos perspectivas: desde la realización de actividades de investigación y desarrollo -I\&D- (Hauknes, 1999) entre empresas y desde la transferencia de información técnica y tecnológica-TITT- (Lagendijk \& Charles, 1999; Nadvi, 1999; Black et al., 2004) para la generación de actividades de I\&D\&I propias de la empresa.

\subsection{I\&D dentro de las redes empresariales como determinante de la innovación y la I\&D en las empresas}

Con respecto a la incidencia que tienen la I\&D realizada en el contexto de las redes empresariales, sobre la innovación y las actividades de I\&D en el ámbito de la empresa existe evidencia empírica que lo demuestra. Evangelista et al. (2002) identificaron en cuatro sistemas regionales de innovación en Italia que existen diferencias en cuanto a la relevancia sobre las actividades de I\&D y de no-I\&D a nivel de las empresas. Cassiman \& Veugelers (2006) sugieren que hay complementariedad entre las actividades internas de I\&D y la adquisición de conocimiento externo con las actividades de innovación, pero que el grado de complementariedad es sensible a otros elementos del ambiente estratégico de la empresa. En relación con la I\&D básica, también plantean la importancia de las universidades y centros de investigación como fuente de información para los procesos de innovación como una variable contextual que afecta la complementariedad entre actividades de innovación interna y externa.

Johansson y Karlsson (2007) encuentran positiva relación entre las actividades I\&D y el desempeño de las empresas en función de la diversidad regional de las exportaciones, lo que implica la introducción de innovaciones de proceso y de producto al nivel de las empresas. Con base en lo anterior se pueden plantear las siguientes hipótesis:

H1: Los vínculos en actividades de I\&D entre las empresas y las instituciones inciden positivamente en la innovación 
(producto, proceso, administrativas) de las propias empresas.

H2: Los vínculos en actividades de I\&D entre las empresas y las instituciones inciden positivamente en las actividades de I\&D (input para la innovación) de las propias empresas.

\subsection{Transferencia de información técnica y tecnológica - TITT - como determinante de la innovación y la I\&D en las empresas}

En los intercambios dentro de las redes empresariales, la TITT es fundamental en la difusión y absorción de innovaciones (Banyte \& Salickaite, 2008) y por lo tanto en el desempeño innovador de la empresa (Evangelista et al, 1997; Capello, 2002; Arvanitis et al., 2005 y 2007; Lin \& Chen, 2006). Además contribuye al incremento de las actividades de I\&D y la obtención de innovaciones propias y disminuyen la dependencia de ideas y tecnologías desarrolladas por otros (Rammer \& Schmiele, 2007). Son variados los mecanismos que se dan para la TITT, en los que se resalta el carácter formal e informal de este proceso (Allen et al., 2007) y así mismo la característica de que el conocimiento puede ser incorporado o desincorporado (OCDE \& EUROSTAT, 2005). Desde el punto de vista formal e informal de la TITT hay en la literatura alusión a los intercambios que suceden entre el personal (vía estructuras formales o informales, Allen et al., 2007) de las empresas y de las instituciones involucradas en redes, a lo que también se ha denominado como capital relacional (Capello, 2002; Capello \& Faggian, 2005). En la perspectiva del conocimiento incorporado y desincorporado, la TITT ha sido estudiada como adquisición de tecnología (Bin, 2008) o como capacidad de absorción de tecnología (Fabrizio, 2009). No obstante importa considerar que la TITT no siempre es producto de transacciones comerciales donde hay un comprador y un vendedor de tecnología, ella ocurre mediante el uso de estrategias diversas como proyectos conjuntos de investigación (o de desarrollo de tecnología), proyectos de capacitación conjunta (Arvanitis et al., 2005) o contratos de apoyo mutuo para la innovación en las empresas $^{2}$ donde las instituciones públicas tienen un importante rol. Igualmente se encuentra la TITT a través del uso conjunto de infraestructura técnica o del uso conjunto de laboratorios, entre otros (Filatochev et al., 2003; Arvanitis et al., 2005, p. 18).

Hay trabajos empíricos que ilustran como la TITT entre empresas que operan en redes localizadas influencian la innovación y las actividades de I\&D propias de la empresa. Capello (2002) encontró

2 En Colombia muchos desarrollos tecnológicos realizados en las empresas han sido producto de la cofinanciación entre universidades y centros de desarrollo tecnológico, las empresas e instituciones de financiamiento estatales, mediante acuerdos de cooperación en los que se obtienen capacidades técnicas y tecnológicas para los actores involucrados. Estadísticas de estos trabajos pueden verse en www.colciencias.gov.co 
evidencia de los efectos de los derrames (spillovers) de conocimiento científico (que es generado por universidades $y$ centros de I\&D) sobre las actividades de innovación en las empresas tanto en las áreas urbanas y no urbanas así como en los distritos.

En particular estos conocimientos son más apreciados por las pequeñas firmas en las áreas no urbanas, situación que se explica por el hecho de que los centros de investigación públicos y privados en estas zonas inician sus investigaciones en la mayoría de los sectores especializados de la zona y por lo tanto esto se constituye en un canal para la adquisición de conocimiento especializado por parte de las empresas. Lin \& Chen, (2006) estudiando la integración de conocimiento en redes para la innovación de productos encontraron que la integración de conocimiento es una variable mediadora entre la orientación al mercado, recursos complementarios e información compartida, variables que según ellos influencian la innovación de producto. Particularmente evidenciaron que la integración de conocimiento influencia positivamente el proceso de desarrollo de nuevos productos en el contexto de redes de industrias. Martínez \& Céspedes (2006) estudiando dos cluster españoles dan evidencia de que las relaciones de las empresas con las asociaciones regionales son utilizadas por las empresas para obtener conocimiento que contribuya con sus capacidades, no obstante no tener relación significativa con la innovación concretamente. Knudsen (2007) estudiando la naturaleza y relativa importancia de las relaciones inter-firmas sobre el éxito en el desarrollo de nuevos produc- tos indica una mayor frecuencia en la participación de los clientes en los esfuerzos de desarrollo. También encuentra que hay una tendencia de las empresas a aliarse con otras de su mismo sector industrial, frente a lo cual anota el peligro que esto envuelve en el sentido de que el conocimiento aportado es similar y por lo tanto afecta la posibilidad de desarrollar innovaciones radicales en productos. Con base en lo anterior se pueden expresar las siguientes hipótesis:

H3: La transferencia de información técnica y tecnológica entre las empresas y las instituciones, incide positivamente en la innovación (producto, proceso, administrativas) de las empresas.

H4: La transferencia de información técnica y tecnológica entre las empresas y las instituciones, incide positivamente en las actividades de I\&D (input para la innovación) de las empresas.

\section{Redes empresariales e innovación en el sector textil}

Al observar los resultados de la Tabla 1, se puede observar que en relación con la variable innovación, el $38 \%$ de las empresas pertenecientes al cluster han realizado innovación (producto, proceso y administrativo), pero solo el $10 \%$ de ellas indicaron haber tenido vínculos para I\&D y apenas el $5 \%$ realizaron TITT en función de la innovación. Con relación a la variable actividades de I\&D, el $24 \%$ de las empresas manifestaron haberlas realizado, no obstante los vínculos con otras empresas e instituciones para I\&D y TITT en función de la I\&D de las propias empresas son bastante bajos, apenas el $2 \%$ y $3 \%$ respectivamente. 


\section{Tabla 1}

Contingencia entre las variables del estudio

\begin{tabular}{|c|c|c|c|c|c|c|c|}
\hline \multicolumn{2}{|l|}{ Variables } & \multicolumn{3}{|c|}{ Vínculos de I\&D } & \multicolumn{3}{|c|}{$\begin{array}{c}\text { Transferencia Técnica } \\
\text { y Tecnológica }\end{array}$} \\
\hline & & Total & No & Sí & Total & No & Sí \\
\hline & No & $62 \%$ & $59 \%$ & $3 \%$ & $62 \%$ & $61 \%$ & $1 \%$ \\
\hline \multirow[t]{2}{*}{ Innovación } & Sí & $38 \%$ & $28 \%$ & $10 \%$ & $38 \%$ & $33 \%$ & $5 \%$ \\
\hline & Total & $100 \%$ & $87 \%$ & $13 \%$ & $100 \%$ & $94 \%$ & $6 \%$ \\
\hline \multirow[t]{3}{*}{ Actividades de I\&D } & No & $76 \%$ & $65 \%$ & $11 \%$ & $76 \%$ & $73 \%$ & $3 \%$ \\
\hline & Sí & $24 \%$ & $22 \%$ & $2 \%$ & $24 \%$ & $21 \%$ & $3 \%$ \\
\hline & Total & $100 \%$ & $87 \%$ & $13 \%$ & $100 \%$ & $94 \%$ & $6 \%$ \\
\hline
\end{tabular}

Fuente: Elaboración propia.

Los resultados dan evidencia, por un lado, de la poca capacidad de las empresas para desarrollar innovaciones y actividades de I\&D que puede ser explicado por la estructura productiva de la red, compuesta en su mayoría por micro y pequeñas empresas cuyos recursos son bastante limitados para invertir en este tipo de procesos y por otro lado que la mayoría de las empresas medianas y las pocas grandes han enfocado su negocio hacia el sistema maquila cuya exigencia en la innovación es bastante reducida y en el mejor de los casos apunta a la eficiencia en los procesos productivos que se expresan básicamente en innovaciones incrementales.

Por otro lado las datos muestran una baja propensión de las empresas a establecer vínculos para actividades de I\&D y para la TITT en función de la innovación y la I\&D en las empresas, lo que sugiere que las empresas tienden a desarrollar dichas actividades de manera independiente. Una explicación para esto puede verse en los hallazgos de Mahemba \& De Bruijn (2003) quienes plantean que las PYMEs en Tanzania no son conscientes de las oportunidades que el medio externo les ofrece tales como colaboración con institutos de investigación, universidades, centros tecnológicos y el gobierno.

No obstante los resultados de la tabla anterior, los casos donde se evidenció que se desarrollaron vínculos para actividades I\&D y TITT en función de la innovación y la I\&D en la empresa permitieron establecer la incidencia de las primeras variables sobre las segundas tala como se expresó en las hipótesis, para ello se desarrolló un análisis de regresión logísti$\mathrm{ca}^{3}$ bajo el método stepwise 4 (Jensen et al., 2007). Los modelos de regresión se contrastaron mediante los incrementos del pseudo-coeficiente de determinación (R2) (Gujarati, 2004).

Para establecer la bondad de los modelos se utilizó el test hosmer lemeshow ${ }^{5}$ y se realizó un análisis de corre- 
laciones estimadas. Con base en ellos se encontró que los parámetros de las variables independientes se ajustan en el modelo, y por tanto explican significativamente $(p$ value $>0,1)$ la incidencia sobre la innovación. Además el análisis de correlaciones estimadas, identificó significativamente $(p$ value $<0,05)$ la no existencia de correlación entre las variables explicativas.

A partir de los modelos de regresión logística se hallaron evidencias para establecer que los vínculos entre empre- sas para desarrollar actividades de I\&D y TITT inciden positivamente sobre la innovación, específicamente la variable vínculos para actividades de I\&D incide significativamente $(p$ value $<0,001)$ sobre la innovación de la empresa, se identifica también que la TITT incide significativamente $(p$ value $<0,05)$ sobre la innovación, lo cual permite aceptar las hipótesis uno y dos (Tabla 2).

Asimismo por medio del modelo es posible identificar que no existe evidencia

\section{Tabla 2}

Incidencia de los vínculos entre empresas para actividades I\&D y de la TITT sobre la innovación en la empresa

\begin{tabular}{|c|c|c|c|c|c|c|}
\hline \multirow[t]{2}{*}{ Variables Predictivas } & \multicolumn{2}{|c|}{ Modelo 1} & \multicolumn{2}{|c|}{ Modelo 2} & \multicolumn{2}{|c|}{ Modelo 3} \\
\hline & B & $\operatorname{Exp}(\beta)$ & B & $\operatorname{Exp}(\beta)$ & B & $\operatorname{Exp}(\beta)$ \\
\hline C & $-7.70 \mathrm{E}-02$ & $9.26 \mathrm{E}-01$ & $-1.99 \mathrm{E}-01$ & 8.19E-01 & $-2.99 \mathrm{E}-01$ & 7.42E-01 \\
\hline Tamaño empresa & $-1.46 \mathrm{E}-04$ & $1.00 \mathrm{E}+00$ & $-3.02 \mathrm{E}-04$ & $1.00 \mathrm{E}+00$ & $1.16 \mathrm{E}-04$ & $1.00 \mathrm{E}+00$ \\
\hline Distribuidores (clientes) & 2.85E-01 & $3.81 \mathrm{E}-01^{* * *}$ & $1.80 \mathrm{E}-01$ & $2.54 \mathrm{E}-01^{* \star *}$ & $-7.45 \mathrm{E}-01$ & 4.75E-01* \\
\hline Proveedores & $-9.66 \mathrm{E}-01$ & $1.33 \mathrm{E}+00$ & $-1.37 \mathrm{E}+00$ & $1.20 \mathrm{E}+00$ & 4.61E-01 & $1.59 \mathrm{E}+00$ \\
\hline Vínculos para I\&D & & & $2.30 \mathrm{E}+00$ & $9.95 \mathrm{E}+00^{\star * *}$ & & \\
\hline TITT & & & & & $1.66 \mathrm{E}+00$ & $5.25 \mathrm{E}+00^{*}$ \\
\hline McFadden R-squared & \multicolumn{2}{|c|}{0.046} & \multicolumn{2}{|c|}{0.133} & \multicolumn{2}{|c|}{0.069} \\
\hline Prob (Hosmer lemeshow) & \multicolumn{2}{|c|}{0.320} & \multicolumn{2}{|c|}{0.212} & \multicolumn{2}{|c|}{0.259} \\
\hline * $\quad p<0,05$ & & & & & & \\
\hline ** $\quad p<0,01$ & & & & & & \\
\hline$* * * \quad p<0,001$ & & & & & & \\
\hline
\end{tabular}

Fuente: Elaboración propia.

3 Becheik et al. (2006) hace una 'revisión sistemática de la literatura' de los estudios empíricos sobre innovación en el que describe las técnicas de análisis de datos frecuentemente utilizadas, allí el modelo logit se ha utilizado en un $12 \%$ (p. 649).

4 Se ingresan paso a paso diferentes tipos de variables con el fin de comparar el modelo por medio del pseudo-coeficiente de determinación.

5 El test permite identificar que los parámetros de las variables independientes se ajustan en la interpretación del modelo, contrastándolos a un nivel de significancia $p$ value $>0.001$. 
para establecer que los vínculos entre empresas para actividades de I\&D y TITT inciden positivamente sobre las actividades de I\&D en las empresas, dado que los modelos no son significativos ( $p$ value $>0,1)$, por tanto no se aceptan las hipótesis 3 y 4 (Tabla 3 ).

Además de las contrastaciones de hipótesis los modelos permiten identificar otros hallazgos relevantes: El primero se relaciona con el modelo que presenta un mayor pseudo coeficiente de determinación (Gujarati, 2004), teniendo en cuenta que ninguno de los modelos relacionados con las actividades de I\&D en la empresa presenta significancia ( $p$ value $>0,10$ ) sólo se realiza el análisis con los modelos relacionados con la innovación en la empresa, esto permite identificar que la variable que mejor explica la variación de la innovación en ambientes de redes empresariales locales son los vínculos para actividades de I\&D. El segundo hallazgo relevante permite identificar que el eslabón de clientes presenta incidencia positiva y significativa sobre la innovación y las actividades de I\&D en la empresa ( $p$ value $<0,001$ y $p$ value $<0,01$ respectivamente) cuando está presente la variable vínculos para actividades de I\&D, caso contrario sucede con el mismo eslabón que presenta incidencia negativa y significativa sobre la innovación y las actividades de I\&D en la empresa ( $p$ value $<0,05$ y $p$ value $<0,01$ respectivamente) cuando está presente la variable TITT. Por último se halló que el eslabón de proveedores no incide significativamente ( $p$ value $=$ $0,05)$ sobre la innovación y las actividades de I\&D en la empresa.

Por último se desarrolla un análisis complementario, con el fin de establecer qué tanto inciden los vínculos sobre las actividades de I\&D e innovación en la em-

\section{Tabla 3}

Incidencia de los vínculos entre empresas para actividades I\&D y de la TITT sobre las actividades de I\&D en la empresa

\begin{tabular}{|c|c|c|c|c|c|c|}
\hline \multirow{2}{*}{$\begin{array}{l}\text { Variables } \\
\text { predictoras }\end{array}$} & \multicolumn{2}{|c|}{ Modelo 1} & \multicolumn{2}{|c|}{ Modelo 2} & \multicolumn{2}{|c|}{ Modelo 3} \\
\hline & B & $\operatorname{Exp}(\beta)$ & $B$ & $\operatorname{Exp}(\beta)$ & $\mathrm{B}$ & $\operatorname{Exp}(\beta)$ \\
\hline C & $-5.98 \mathrm{E}-01$ & $5.50 \mathrm{E}-01^{* *}$ & $-5.66 \mathrm{E}-01$ & $5.68 \mathrm{E}-01$ & $-6.65 \mathrm{E}-01$ & $5.15 \mathrm{E}-01^{* *}$ \\
\hline Tamaño empresa & $-3.07 \mathrm{E}-02$ & $9.70 \mathrm{E}-01$ & $-2.94 \mathrm{E}-02$ & $9.71 \mathrm{E}-01$ & $-2.89 \mathrm{E}-02$ & $9.71 \mathrm{E}-01$ \\
\hline Distribuidores (clientes) & $1.05 \mathrm{E}-01$ & $3.98 \mathrm{E}-01^{* *}$ & $1.42 \mathrm{E}-01$ & 4.23E- $01^{* *}$ & $-8.63 E-01$ & $4.22 \mathrm{E}-01^{* *}$ \\
\hline Proveedores & $-9.22 \mathrm{E}-01$ & $1.11 \mathrm{E}+00$ & $-8.61 \mathrm{E}-01$ & $1.15 E+00$ & $1.50 \mathrm{E}-01$ & $1.16 \mathrm{E}+00$ \\
\hline Vínculos para I\&D & & & $4.96 \mathrm{E}+01$ & $5.09 \mathrm{E}+01^{* * *}$ & & \\
\hline TITT & & & & & $9.25 \mathrm{E}-01$ & $1.40 \mathrm{E}-01^{*}$ \\
\hline McFadden R-squared & \multicolumn{2}{|c|}{0.048} & \multicolumn{2}{|c|}{0.054} & \multicolumn{2}{|c|}{0.049} \\
\hline Prob (Hosmer lemeshow) & \multicolumn{2}{|c|}{0.928} & \multicolumn{2}{|c|}{0.557} & \multicolumn{2}{|c|}{0.880} \\
\hline * $\quad p<0,05$ & & & & & & \\
\hline ** $\quad p<0,01$ & & & & & & \\
\hline${ }^{* * *} \quad p<0,001$ & & & & & & \\
\hline
\end{tabular}

Fuente: Elaboración propia. 
presa, para esto se desarrolla análisis de efectos marginales (ver Tabla 4) el cual, permite evidenciar que la existencia de vínculos para actividades de I\&D en red incrementan la probabilidad de generar innovación en la empresa en un 49\%, e incrementan la probabilidad de generar actividades de I\&D en la empresa en un $80 \%$, igualmente la existencia de vínculos para la TITT incrementa la probabilidad de generar actividades de I\&D en la empresa en un $20 \%$, e incrementan la probabilidad de generar innovación en la empresa en un $27 \%$. Aspectos que permiten evidenciar el impacto altamente significativo que generan los vínculos entre empresas para generar actividades de I\&D\&I en la propia empresa (ver Tabla 4).

Estos resultados permiten hallar evidencia que las relaciones entre empresas y otras instituciones inciden positivamente en la innovación y las actividades de I\&D en las empresas. En forma similar (Fabrizio, 2009) anota que la investigación interna junto con la colaboración externa contribuye a la investigación para la innovación en términos del ritmo de la innovación. En particular los vínculos entre empresas para actividades de I\&D y la TITT (spillover de conocimientos o capital relacional) tienen una incidencia positiva sobre la innovación en la empresa, hecho que también ha sido constatado por otros investigadores, en este sentido Capello (2002) concluye que los mecanismos de cooperación local, las sinergias y la producción de conocimiento son un aspecto importante en la capacidad innovadora de las firmas. Fabrizio (2009) plantea que las conexiones a fuentes de conocimiento externo facilitan los procesos de solución, que las conexiones con científicos externos hacen más eficiente la búsqueda de conocimiento, y agrega que el impacto de las estructuras de red sobre los resultados en la innovación es común en características como la capacidad de absorción de la firma. Eraydin \& AmartliKöroglu (2005) concluyen que los vínculos con otras firmas, instituciones y organizaciones tienen una positiva influencia sobre la capacidad innovadora de la firma tal como lo sugiere la literatura.

La realización de actividades de I\&D son un input para la innovación, bajo esta perspectiva se planteó en el estudio que la realización de este tipo de actividades en asociación con otras empresas e instituciones así como la TITT incidieran positivamente la I\&D dentro de la empresa, al respecto los resultados encontrados no permiten concluir que efectivamente esto suceda, a pesar de ser reconocido en la literatura. Podrían plantear-

Tabla 4

Efectos marginales de las variables predictoras sobre las actividades de I\&D y la innovación en la empresa.

\begin{tabular}{lcc}
\hline \multicolumn{1}{c}{ Variables } & $\begin{array}{c}\text { Actividades de I\&D } \\
\text { en la empresa (dy/dx) }\end{array}$ & $\begin{array}{c}\text { Innovación en la empresa } \\
(\mathbf{d y} / \mathbf{d x})\end{array}$ \\
\hline Vínculos para I\&D & $80 \%^{* * *}$ & $49 \%^{* * *}$ \\
TITT & $20 \%^{*}$ & $27 \%^{* * *}$ \\
\hline${ }^{* * *} \mathrm{p}$ value $<0,001 .{ }^{* *} \mathrm{p}$ value $<0,01$. & $* \mathrm{p}$ value $<0,05$ & \\
Fuente: resultados de la investigación. & &
\end{tabular}


se algunas razones para este hallazgo, en primer lugar la tendencia encontrada en estas empresas a actuar al margen de las oportunidades que el medio externo ofrece (Mahemba \& De Bruijn, 2003), otro aspecto está relacionado con que las pequeñas empresas son menos proclives a la innovación y por lo tanto desarrollan menos actividades de I\&D, situación que pone en evidencia Evangelista et al. (1997) al estudiar las relaciones entre innovación y tamaño de la firma. Otro elemento que puede ofrecer una explicación radica en que las empresas del estudio pertenecen a un sector tradicional de la economía cuyo dinamismo tecnológico es limitado, y por su tamaño están muy centradas en el mercado local lo que les determina bajos niveles de actividades formales de innovación (Beneito, 2002).

Con respecto a la importancia de las relaciones empresariales dentro de la cadena de producción (proveedor - fabrica cliente) este trabajo ha permitido establecer que los clientes tienen una mayor incidencia en los vínculos para actividades de I\&D y TITT en función de la innovación de los fabricantes, lo que significa que estos dos eslabones son más proclives a trabajar relacionados. Los resultados de los proveedores son consistentes con la literatura, pero los relacionados con los clientes no. (Nadvi, 1999) encontró positivas correlaciones analizando la colaboración con compradores, clientes y subcontratistas, y Knudsen (2007) evidenció la importancia de los clientes en la innovación de producto. La situación presentada en este estudio puede explicarse por la composición de las empresas, que en su mayoría son micro y pequeñas unidades y con una tendencia a trabajar en solitario. Por otro lado la expectativa de que las empresas de mayor tamaño impulsaran las relaciones tampoco se dio, en este sentido la explicación estaría en el número de empresas dentro de la red (apenas 3\% incluyendo grande y mediana) y además todas tienen la característica de ser maquilas, por lo que sus relaciones están sobre todo por fuera de la localidad.

\section{Conclusiones}

Los resultados permiten concluir en primer lugar que las empresas del cluster textil - confección presentan una baja propensión para establecer vínculos entre empresas para el desarrollo de actividades de I\&D y para la TITT, aspecto que incide en la baja proporción de empresas que realizan actividades de I\&D e innovación dentro de la empresa, lo cual es un indicador preocupante en materia de competitividad del sector.

En segundo lugar se evidencia que la existencia de vínculos entre empresas para realizar actividades de I\&D y TITT inciden directamente sobre la capacidad innovadora de la empresa, y específicamente permiten desarrollar las actividades de I\&D\&I en la propia empresa, en mayor proporción que aquellas que no desarrollan vínculos con otras empresas e instituciones (capital relacional).

En este sentido, preocupa el hecho que la relación entre empresa y proveedores no genere resultados hacia la innovación, evidenciándose que a mayor relación con los proveedores menor será la capacidad innovadora de la empresa, aspecto que no es coherente con la literatura estudiada ni con otros contextos similares, por tanto se hace necesario desa- 
rrollar otras investigaciones encaminadas a estudiar los vínculos entre proveedores y empresa.

Por último, teniendo en cuenta la evidencia encontrada en el estudio, donde pocas empresas tienen vínculos entre ellas y con otras instituciones, se hace necesario desarrollar políticas sectoriales que fortalezcan el capital relacional empresarial, en la medida que éste es un impulsor de las actividades de I\&D\&I de la empresa.

\section{Referencias bibliográficas}

Alecke, Bjorn; Alsleben, Christoph; Scharr, Frank y Gerhard, Untiedt (2006). Are there really high-tech clusters? The geographic concentration of German manufacturing industries and its determinants. En: Annals Regional Science, No. 40, US, George Mason University (19-42).

Allen, James; Andrew James, y Phil Gamlen (2007) Formal versus informal knowledge networks in R\&D: A case study using social network analysis. En: R\&D Management, vol.37, No. 3 UK, University of Manchester (179 - 196).

Alonso-Villar, Olga; Chamorro, José-María y Xulia, Gonzáles (2004). Agglomeration economies in manufacturing industries: the case of Spain. En: Applied Economics, No. 36, UK, University of Warwick (2103-2116).

Arvanitis, Spyros; Kubli, Ursina; Sydow Nora y Martin, Wörter (2005). Knowledge and technology transfer (KTT) activities between universities and firms in Switzerland: the main facts. En: KOF Working Paper, No. 115, Zurich, KOF.

Arvanitis, Spyros; Kubli, Ursina; Sydow Nora y Martin, Wörter (2007). Knowledge and technology transfer (KTT) activi- ties between universities and firms in Switzerland - the main facts: an empirical analysis based on firm-level data. En: The Icfai Journal of Knowledge Management, Vol.5, No.6, UK, Teleos (17-75).

Banco Interamericano de Desarrollo -BID(2002). Vinculaciones empresariales en la industria de la confección. USA.

Banyte, Jüratè y Rüta, Salickaite (2008). Successful diffusion and adoption of innovation as a means to increase competitiveness of enterprises. En: Engineering economics, Vol.1, No.56. LT, Kauno Technologijos Universitetas (48-56).

Baptista, Rui y Gavin Swann (1998). Do firms in clusters innovate more? En: Research Policy, No. 27, US, Carnegie Mellon University (525-540).

Beaudry, Catherine y Stefano, Breschi (2003). Are firms in clusters really more innovative?. En: Economy Innovation New Technology, Vol.12, No.4, IT, Salvatore Cognetti de Martiis (325-342).

Becerra, Fredy (2008). Las redes empresariales y la dinámica de la empresa: aproximación teórica. En: Innovar, Vol.18, No.32, CO, Universidad Nacional de Colombia (27-45).

Becerra, Fredy y Claudia Álvarez (2011). El talento humano y la innovación empresarial en el contexto de las redes empresariales: el cluster de prendas de vestir en Caldas - Colombia. En: Estudios Gerenciales, Vol.27, No.119 CO, Universidad ICESI (209-234).

Becerra, Fredy y Julia, Naranjo (2008) La innovación tecnológica en el contexto de los clusters regionales. En: Cuadernos de administración. Vol. 21, No. 37, CO, Pontificia Universidad Javeriana (133-159).

Becheikh, Nizar; Landry, Réjean y Nabil, Amara (2006). Lessons from innovation 
empirical studies in the manufacturing sector: a systematic review of the literature from 1993-2003. En: Technovation, No.26, CA, University of Ottawa $(644-664)$.

Belussi, F. y Pilotti, L. (2002): "Knowledge creation, learning and innovation in italian industrial districts En: Geografiska Annaler No. 84, SW, Swedish Society for Anthropology and Geography $(125-139)$.

Beneito, Pilar (2002). Technological patterns among Spanish manufacturing firms. En: Entrepreneurship \& Regional Development, No.14, UK, Robert Gordon Univeristy $(89-115)$.

Bin, Guo (2008). Technology acquisition channels and industry performance: an industry-level analysis of Chinese large- and medium-size manufacturing enterprises. En: Research Policy, No.37, US, Carnegie Mellon University $(194-209)$.

Black, Geoffrey; Chucrch, Jhon y Donald, Holley (2004). Empirical estimation of agglomeration economies associated with research facilities. En: Atlantic Economic Journal, Vol.32, No.4, US, International Atlantic economic society $(320-328)$.

Callejón, María (1998). Concentración geográfica de la industria y economías de aglomeración. Universidad de Barcelona Facultad de Ciencias Económicas y Empresariales. ES, Universidad de Barcelona, Free press.

Camisón, César; Lapiedra, Rafael; Segarra, Mercedes y Montse Boronat (2003). Marco conceptual de la relación entre innovación y tamaño organizativo. En: Tribuna de debate. No.19, ES, Madritd, Free press.

Caniëls, Marjolein y Henny Romijn (2005). Agglomeration advantages and capability building in industrial clusters: the missing link. En: The Journal of Development Studies, Vol.39, No. 3, UK, London School of economics (129 - 154).

Capello, Roberta (2002). Spatial and sectoral characteristics of relational capital in innovation activity. En: European Planning Studies, Vol.10, No.2, BE, Catholic University of Leuven (177 200).

Capello, Robertta y Alessandra, Faggian (2005). Collective learning and relational capital in Local innovation processes. En: Regional Studies, Vol.39, No.1, NL, Radboud University Nijmegen (75-87).

Cardona, Marleny \& Carlos, Cano (2004). Un acercamiento sectorial y regional del ciclo de vida de las firmas colombianas a través de un modelo datos de panel. Disponible en: http://www.eumed.net/cursecon/ecolat $/ \mathrm{co} /$, consultada en: octubre de 2010.

Cardona, Marleny, Cano, Carlos, Ramírez, Carlos y Jahir, Gutiérrez (2006). Mundos de producción de las PyMEs en Colombia: Una aproximación desde las tecnologías, la organización y las políticas industriales. En: Archivos de economía. CO, Departamento Nacional de Planeación.

Cassiman, Bruno y Reinhilde Veugelers (2006). In search of complementarity in innovation strategy: internal R\&D and external knowledge acquisition. En: Management Science, Vol.52, No.1, US, University of Pennsylvania $(68-82)$.

Consejo Privado de Competitividad CPC (2008) Informe de competitividad 2008 - 2009. Ruta a la prosperidad colectiva. CO.

Damanpour, Fariborz (1987). The adoption of technological, administrative, and ancillary innovations: impact of organi- 
zational factors. En: Journal of Management, US, No.13, Southern Management Association (675 - 688).

Damanpour, Fariborz (1991): “Organizational Innovation: A meta-analysis of effects of determinants and moderators". En:

Academy of Management Journal, No.34, US, Academy of Management, (555 - 590).

Damanpour, Fariborz y Santhi Gopalakrishnan (1998). Theories of organizational structure and innovation adoption: the role of environmental change. En: Journal of Engineering and Technology Management, Vol.15, No.1, CA, Simon Fraser University (1-24).

Damanpour, Fariborz y Santhi Gopalakrishnan (2001). The dynamics of the adoption of product and process innovations in organizations. En: Journal of Management Studies, Vol.38, No.1, US, Babson College (45-65).

Damanpour, Fariborz; Szabat, Kathryn y William Evan (1989). The relationship between types of innovation and organizational performance. En: Journal of Management Studies, Vol.26, No.6, US, Babson College (587 602).

Departamento Nacional de Planeación -DNP(2010). Generalidades de la cadena productiva. $\mathrm{CO}$.

Departamento Nacional de Planeación -DNP(2007). Agenda interna para la productividad y la competitividad: Documento sectorial, algodón, fibra, textil y confecciones. $\mathrm{CO}$.

Edwards, Tim (2003). Innovation and organizational change: developments towards an interactive process perspective. En: Technology Analysis \& Strategic Management, Vol.12, No.4, UK, Manchester Business School, (445 - 464).

Eraydin, Ayda y Bilge Armatli-Köroglu (2005). Innovation, networking and the new industrial clusters: the characteristics of networks and local innovation capabilities in the Turkish industrial clusters. En: Entrepreneurship and regional development, No.17, UK, Robert Gordon University (237-266).

Evangelista, Rinaldo; Perani, Giulio; Rapiti, Fabio y Daniele Archibugi (1997). Nature and impact of innovation in manufacturing industry: some evidence from the Italian innovation survey. En: Research Policy, No.26, US, Carnegie Mellon University (521 - 536).

Evangelista, Rinaldo; lammarino, Simona; Mastrostefano, Valeria y Alberto Silvani (2002). Looking for regional systems of innovation: evidence from the Italian innovation survey. En: Regional Studies, Vol.36, No.2, NL, Radboud University Nijmegen, (173-186).

Fabrizio, Kira (2009). Absorptive capacity and the search for innovation. En: Research Policy, No. 38, US, Carnegie Mellon University (255 - 267).

Feldman, Maryann y David Audretsch (1999). Innovation in cities: science-based diversity, specialization and localized competition. En: European Economic Review, No. 43, NL, Universiteit Maastricht, (409-429).

Feser, Edward y Edward Bergman (2000). National industry cluster templates: a framework for applied regional cluster analysis. En: Regional Studies, Vol. 34, No.1, NL, Radboud University Nijmegen, $(1-19)$.

Filatochev, Igor; Piga, Claudio y Natalya Dyomina (2003). Network positioning and R\&D activity: a study of Italian groups. En: R and D Management, Vol. 33, No. 1, UK, University of Manchester (37-48).

Gujarati, Damodar (2004). Econometría. MX, McGraw Hill.

Hage, James (1999). Organizational innovation and organizational change. En: 
Annual Review of Sociology, Vol. 25, US, Stanford University (597 $622)$.

Hauknes, Johan (1999). Norwegian input-output clusters and innovation patterns. En: OCDE, Boosting innovation: The cluster approach, FR (61 - 90).

Hervás, Jose and Juan Dalmau. (2006). How to measure IC in clusters: empirical evidence. Journal of Intellectual Capital, Vol. 7 No. 3, UK, Teleos (354 - 380).

Hu, Tai-shan; Lin, Chien-yuang. Su-Li Chang (2005). Role of interaction between technological communities and industrial clustering in innovative activity: the case of Hsinchu district, Taiwan. En: Urban Studies, Vol. 42, No. 7, UK, University of Glasgow (11391160).

Jamrog, Jay; Vickers, Mark y Donna Bear (2006). Building and sustaining a culture that supports innovation. En: Human Resources Planning, Vol. 29, No.3, US, Human Resource Planning Society $(9-19)$.

Jaskyte, Kristina (2005). Transformational leadership, organizational culture, and innovativeness in nonprofit organizations. En: Nonprofit Management \& Leadership, Vol. 15 No. 2, US, Case Western University $(153-168)$.

Jensen, Robert (1996). Social issues in spatial economics. En: International Journal of Social Economics, Vol. 23, No. 4/5/6, UK, University of Hull (297 309).

Johansson, Sara y Charlie Karlsson (2007). R\&D accessibility and regional export diversity. En: Annals Regional Science, Vol. 41, SE, Jönköping University (501-523).

Knudsen, Mette (2007). The relative importance of interfirm relationships and knowledge transfer for new product development success. En: The Journal of Product Innovation Manage- ment, Vol. 24, US, Temple University (117-138).

Lagendijk, Arnoud y David Charles (1999). Clustering as a new growth strategy for regional economies? A discussion of new forms of regional industrial policy in the United Kingdom. En: OCDE, Boosting Innovation: The Cluster Approach, FR (127 - 154).

Lin, Bou-Wen y Chung-Jen Chen (2006). Fostering product innovation in industry networks: the mediating role of knowledge integration. En: The International journal of Human Resource Management, Vol. 17, No.1, UK, University of Cardiff, (155-173).

López, Giovanni (2006). Perspectivas para el análisis de la innovación: un recorrido por la teoría. En: Cuadernos de Administración, Vol. 19 No. 31, CO, Pontificia Universidad Javeriana (243 -273 ).

Mahemba, Christopher y Erik De Bruijn (2003). Innovation activities by small and medium-sized manufacturing enterprises in Tanzania. En: Creativity and Innovation Management, Vol. 12 No. 3, NL, University of Twente (162 - 173).

Martínez, Javier y José Céspedes (2006). Generación y difusión de la innovación en distritos industriales. En: Tribuna de debate No. 36. ES, Madri+d, Free press.

Ministerio de Comercio, Industria y Turismo -MCIT- (2009). Desarrollando sectores de clase mundial en Colombia. Sector Textil, Confección, Diseño y Moda. CO.

Mella, José; López, Asunción y Coro Yrigoyen (2005). Crecimiento económico y convergencia urbana en España. ES.

Muscio, Alessandro (2006). Patterns of innovation in industrial districts: an empirical analysis. En: Industry and Innovation, Vol. 13, No. 3, DK, Copenhagen Business School (291-312). 
Nadvi, Khalid (1999). Collective efficiency and collective failure: the response of the Sialkot surgical instrument cluster to global quality pressures. En: World Development, Vol. 27, No. 9, CA, McGill University (1605-1626).

Nassimbeni, Guido (2003). Local manufacturing systems and global economy: are they compatible? The case of the Italian eyewear district. En: Journal of Operations Management, Vol. 21, No. 2, US, Arizona State University (151-171).

Novick, Marta y Jorge Carrillo (2007). Eslabonamientos productivos globales y actores locales: debates y experiencias en América Latina. Disponible en: http://docencia.izt.uam.mx/egt/publicaciones/libros/teoría_social/cap12. pdf. consultada en: enero de 2008 ,

OCDE (1999a). Boosting Innovation: the Cluster Approach. FR.

OCDE (1999b). Managing National Innovation Systems. FR.

OCDE/FECYT (2002). Manual de Frascati: propuesta de norma práctica para encuestas de investigación y desarrollo experimental. FR.

OCDE/EUROSTAT (2005). Manual De Oslo: guía para la recogida e interpretación de datos sobre innovación. ES.

Pietrobelli, Carlo y Tatiana Olarte (2002) Enterprise clusters and industrial districts in Colombia's fashion sector. En: European planning studies. Vol.10 No.5 BE, Catholic University of Leuven (541-562).

Power, Dominic y Mats Lundmark (2004). Working through knowledge pools: labour market dynamics, the transference of knowledge and ideas, and indus- trial clusters. En: Urban Studies, Vol. 41 No. 5/6, UK, University of Glasgow, (1025-1044).

Pöyhönen, Aino y Anssi Smedlund (2004). Assessing intellectual capital creation in regional clusters. En: Journal of InteIlectual Capital, Vol. 5, No. 3, UK, Teleos (351-365).

PROEXPORT (2009). Invierta en trabajo. Industria Textil y Confecciones. CO.

Rammer, Christian y Anja Schmiele (2009). Drivers and effects of internationalizing innovation by SMEs. En: The Icfai Journal of Knowledge Management, Vol. 7, No. 2, UK, Teleos. 18-61.

Seufert, Andreas; Von Krogh, George y Andrea Bach (1999). Towards knowledge networking. En: The Icfai Journal of Knowledge Management, Vol. 3 No. 3, UK, Teleos $(180-190)$.

Schumpeter, Joseph (1997). Teoría del desenvolvimiento económico. MX.

Slappendel, C. (1996). Perspectives on innovation in organizations. En: Organization Studies, Vol. 17, No. 1, AU, University of Western (107-129).

Taylor, Alva; Foster-Johnson, Lynn y Richard Harriman (2008). Intra-Firm learning from innovative activity: the role of managers, work context and situated organizational attention. En: Tuck School of Business Working Paper, No. 2008-55, US, Social Science Research Network.

West, Michael y James Farr (990). Innovation and creativity at work. US.

Wolfe, Richard (1994). Organizational innovation: review, critique and suggested research directions. En: Journal of Management Studies, Vo. 31 No. 3, US, Babson College (405-431). 